\title{
THE SPACE OF ISOMETRY COVARIANT TENSOR VALUATIONS
}

\author{
D. HUG, R. SCHNEIDER, AND R. SCHUSTER
}

Dedicated to Professor Viktor Abramovich Zalgaller on the occasion of his 85th birthday

\begin{abstract}
It is known that the basic tensor valuations which, by a result of S. Alesker, span the vector space of tensor-valued, continuous, isometry covariant valuations on convex bodies, are not linearly independent. P. McMullen has discovered linear dependences between these basic valuations and has implicitly raised the question as to whether these are essentially the only ones. The present paper provides a positive answer to this question. The dimension of the vector space of continuous, isometry covariant tensor valuations, of a fixed rank and of a given degree of homogeneity, is explicitly determined. The approach is constructive and permits one to provide a specific basis.
\end{abstract}

\section{$\S 1$. INTRODUCTION}

A function $\varphi$ from the space $\mathcal{K}^{n}$ of convex bodies in Euclidean space $\mathbb{R}^{n}(n \geq 2)$ into some Abelian group is a valuation if it satisfies $\varphi\left(K_{1} \cup K_{2}\right)+\varphi\left(K_{1} \cap K_{2}\right)=\varphi\left(K_{1}\right)+$ $\varphi\left(K_{2}\right)$ whenever $K_{1}, K_{2}, K_{1} \cup K_{2} \in \mathcal{K}^{n}$. The vector space of real valuations on $\mathcal{K}^{n}$ that are continuous (with respect to the Hausdorff metric) and invariant under rigid motions is spanned by the intrinsic volumes and thus has dimension $n+1$. This is the celebrated characterization theorem of Hadwiger, one of the central results of classical convex geometry. Surveys on valuations were given in [15] and [13]. More recently, there are important new developments, beginning with an elegant proof of Hadwiger's theorem by Klain [11], and culminating in the work of S. Alesker. Among the results of Alesker is the proof in [5] of a conjecture of McMullen [12] on the classification of the continuous, translation invariant, real valuations. The space of these valuations is of infinite dimension. On the other hand, Alesker [4] showed the following. If $G$ is a compact subgroup of the orthogonal group acting transitively on the unit sphere of $\mathbb{R}^{n}$, then the space of $G$-invariant, translation invariant, continuous real valuations is finitedimensional. For the cases of the groups $U(n)$ on $\mathbb{C}^{n} \simeq \mathbb{R}^{2 n}$ and $S U(2)$ on $\mathbb{C}^{2} \simeq \mathbb{R}^{4}$, Alesker $[6,7]$ determined these spaces and their dimensions explicitly.

As a natural generalization of the motion invariant real valuations, McMullen [14] introduced isometry covariant tensor valuations, and he formulated the aim to find a characterization of such valuations, under continuity assumptions. To explain this, we denote by $\mathbb{T}^{r}$ the vector space of symmetric tensors of rank $r \in \mathbb{N}_{0}$ (the nonnegative integers) over $\mathbb{R}^{n}$ (we use the scalar product of $\mathbb{R}^{n}$ to identify $\mathbb{R}^{n}$ with its dual space). The symmetric tensor product of symmetric tensors $a, b$ is denoted by $a b$. We write $x^{r}$ for the $r$-fold symmetric tensor product of $x \in \mathbb{R}^{n}$. The normalization is chosen so that $x^{r}=x \otimes \cdots \otimes x$ (with $r$ factors $x$ ). A tensor valuation on $\mathcal{K}^{n}$ is a valuation on $\mathcal{K}^{n}$

2000 Mathematics Subject Classification. Primary 52A20.

Key words and phrases. Convex body, tensor valuation, isometry covariance.

Supported in part by the European Network PHD, FP6 Marie Curie Actions, RTN, Contract MCRN511953. 
with values in $\mathbb{T}:=\bigoplus_{r \in \mathbb{N}_{0}} \mathbb{T}^{r}$. The tensor valuation $\varphi$ is said to be isometry covariant if it has the following two properties: (a) $\varphi(U K)=U(\varphi(K))$ for all $K \in \mathcal{K}^{n}$ and all $U \in O(n)$ (the orthogonal group of $\mathbb{R}^{n}$ ), where $U$ also denotes the canonical extension to $\mathbb{T}$; (b) there is a number $s \in \mathbb{N}$ such that $\varphi$ maps into $\bigoplus_{r=0}^{s} \mathbb{T}^{r}$, and there are functions $\varphi_{j}: \mathcal{K}^{n} \rightarrow \bigoplus_{r=0}^{s} \mathbb{T}^{r}$, for $j=0, \ldots, s$, such that

$$
\varphi(K+t)=\sum_{r=0}^{s} \varphi_{s-r}(K) \frac{t^{r}}{r !},
$$

for $K \in \mathcal{K}^{n}$ and $t \in \mathbb{R}^{n}$. (Thus, $\varphi$ has polynomial behavior. Polynomial valuations were first studied, on polytopes, by Pukhlikov and Khovanskiu [17].) The continuity of such a tensor valuation refers to the natural induced topology on $\bigoplus_{r=0}^{s} \mathbb{T}^{r}$. A tensor valuation taking its values in $\mathbb{T}^{p}$ is said to be of rank $p$. Examples are obtained as follows. If $B^{n}$ denotes the unit ball of $\mathbb{R}^{n}$ and if $\epsilon>0$, then, for $p \in \mathbb{N}_{0}$ and $K \in \mathcal{K}^{n}$,

$$
\int_{K+\epsilon B^{n}} x^{p} \mathrm{~d} x
$$

is a polynomial in $\epsilon$ of degree at most $n+p$. The coefficients of this generalized Steiner polynomial (which were studied in [21]) are isometry covariant, continuous tensor valuations of rank $p$. They span the linear space of all such valuations in the cases where $p=0$ (Hadwiger's theorem) or $p=1$. The latter was proved by Hadwiger and Schneider [10], based on results in $[18,19]$ (since one of the coefficients of the Steiner formula for $p=1$ vanishes identically, the space again has dimension $n+1)$. For $p \geq 2$, however, the coefficients of the Steiner polynomial are not sufficient to span the corresponding space. For obtaining sufficiently many isometry covariant tensor valuations, we need the support measures (or generalized curvature measures) $\Lambda_{0}(K, \cdot), \ldots, \Lambda_{n-1}(K, \cdot)$ of a convex body $K \in \mathcal{K}^{n}$. We briefly recall their definition. Let $\langle\cdot, \cdot\rangle$ be the scalar product and $\|\cdot\|$ the norm in $\mathbb{R}^{n}$. For $x \in \mathbb{R}^{n}$, let $p(K, x)$ denote the metric projection of $x$ to $K$, and put $u(K, x):=(x-p(K, x)) /\|x-p(K, x)\|$ for $x \notin K$. Let $\mathbb{S}^{n-1}$ denote the unit sphere in $\mathbb{R}^{n}$. Then, for any $\epsilon>0$ and Borel set $\eta \subset \Sigma:=\mathbb{R}^{n} \times \mathbb{S}^{n-1}$, the $n$-dimensional Hausdorff measure (volume) $\mathcal{H}^{n}$ of the local parallel set

$$
M_{\epsilon}(K, \eta):=\left\{x \in\left(K+\epsilon B^{n}\right) \backslash K:(p(K, x), u(K, x)) \in \eta\right\}
$$

is a polynomial in $\epsilon$,

$$
\mathcal{H}^{n}\left(M_{\epsilon}(K, \eta)\right)=\sum_{i=0}^{n-1} \epsilon^{n-i} \kappa_{n-i} \Lambda_{i}(K, \eta) .
$$

This defines the support measures. For further information, see [20, Section 4.2] (but observe the different normalization used there) and [22].

For $K \in \mathcal{K}^{n}$ and integers $r, s \geq 0,0 \leq k \leq n-1$, define

$$
\begin{aligned}
\Phi_{n, r, 0}(K) & :=\frac{1}{r !} \int_{K} x^{r} \mathcal{H}^{n}(\mathrm{~d} x), \\
\Phi_{k, r, s}(K) & :=\frac{1}{r ! s !} \frac{\omega_{n-k}}{\omega_{n-k+s}} \int_{\Sigma} x^{r} u^{s} \Lambda_{k}(K, \mathrm{~d}(x, u)),
\end{aligned}
$$

where $\omega_{n}=2 \pi^{n / 2} / \Gamma(n / 2)$ is the surface area of $\mathbb{S}^{n-1}$ (the normalizing factors will have a simplifying effect later). Each function $\Phi_{k, r, s}$ is a continuous, isometry covariant tensor valuation on $\mathcal{K}^{n}$. The same properties are shared by $Q^{l} \Phi_{k, r, s}$ for $l \in \mathbb{N}_{0}$, where $Q$ denotes the (constant) metric tensor defined by $Q(x, y):=\langle x, y\rangle$. We call the functions $\Phi_{k, r, s}$, for $r, s \in \mathbb{N}_{0}$ and either $k \in\{0, \ldots, n-1\}$ or $(k, s)=(n, 0)$, the Minkowski tensors (since for $r+s=0$ they were introduced by Minkowski), and the functions $Q^{l} \Phi_{k, r, s}$, where $l \in \mathbb{N}_{0}$ and $\Phi_{k, r, s}$ is a Minkowski tensor, are called the basic tensor valuations. To simplify the 
sum notation, we put $\Phi_{k, r, s}:=0$ if $k \notin\{0, \ldots, n\}$, or if $r$ or $s$ is not in $\mathbb{N}_{0}$, or if $k=n$ and $s \neq 0$. A function $\Phi_{k, r, s}$ will be called nontrivial if it is a Minkowski tensor.

When McMullen [14] introduced these tensor valuations, he also discovered that they satisfy the relations

$$
2 \pi \sum_{s} s \Phi_{k-r+s, r-s, s}-Q \sum_{s} \Phi_{k-r+s, r-s, s-2}=0
$$

for $k, r \in \mathbb{N}_{0}$. (An analytical proof, different from McMullen's polytope approach, was indicated in [22], based on [21].) McMullen found this to suggest that the characterization problem might not be straightforward, and later in [14] he collected evidence for any solution to be rather complicated. Surprisingly, it turned out that Alesker's work [2] on rotation invariant valuations on convex sets put him in a position to solve McMullen's characterization problem, right after it had been formulated. Alesker showed that the basic tensor valuations $Q^{l} \Phi_{k, r, s}$, with suitable choices of integers $l, k, r, s$, indeed span the vector space of all isometry covariant, continuous tensor valuations of a fixed rank. Based on [2], he announced in [1] and proved in [3] the following theorem.

Theorem 1.1 (Alesker). Let $p \in \mathbb{N}_{0}$, and let $\varphi: \mathcal{K}^{n} \rightarrow \mathbb{T}^{p}$ be a continuous, isometry covariant valuation. Then $\varphi$ is a linear combination, with constant real coefficients, of the basic tensor valuations $Q^{l} \Phi_{k, r, s}$, where $l, k, r, s$ are such that $2 l+r+s=p$.

This is a very remarkable extension of the known results for $p=0$ and $p=1$, but leaves open the determination of a basis and thus of the dimension of the space of continuous, isometry covariant tensor valuations of given rank. The spanning system provided by Alesker's theorem is not linearly independent, by (1.1). The problem of determining all linear dependences between the basic tensor valuations came up soon after McMullen had found (1.1). The main purpose of the present paper is a proof of the fact that the relations (1.1) are essentially (that is, up to multiplications by powers of $Q$ and linear combinations) the only linear dependences between the basic tensor valuations. A precise result is stated in Theorem 2.1. In $\S 3$, we investigate the problem of determining the dimension and a basis of the vector space of continuous, isometry covariant tensor valuations of given rank.

As an example, we mention the simplest new case, the vector space of continuous, isometry covariant tensor valuations of rank two on $\mathcal{K}^{n}$. It has dimension $3 n+1$, and a basis is given by

- $Q \Phi_{j, 0,0}, j=0, \ldots, n$;

- $\Phi_{j, 2,0}, j=0, \ldots, n$;

- $\Phi_{j, 0,2}, j=1, \ldots, n-1$.

This could still be deduced by a direct argument, but it is also a very special consequence of a general result in $\S 3$ (cf. also $\S 4$ ). Since (1.1) yields the formula

$$
\Phi_{k, 0,2}=\frac{1}{4 \pi} Q \Phi_{k, 0,0}-\frac{1}{2} \Phi_{k-1,1,1},
$$

for $k=1, \ldots, n-1$, we can replace $\Phi_{j, 0,2}$, for $j \in\{1, \ldots, n-1\}$, by $\Phi_{j, 1,1}$, for $j \in$ $\{0, \ldots, n-2\}$, in the displayed basis.

For tensor valuations of higher rank, the situation turns out to be considerably more complicated.

Finally in this Introduction, we mention that the Minkowski tensors have also begun to play a role (at least, up to rank two) in the applied sciences, as tools in the morphometry of spatial patterns; see [9, 8], for example. 


\section{$\S 2$. LINEAR DEPENDENCES}

In this section, we prove that the relations between basic tensor valuations discovered by McMullen are essentially the only ones, namely, that any linear relation between basic tensor valuations can be obtained by multiplying relations of the form (1.1) by powers of $Q$ and by taking linear combinations of relations obtained in this way. A more formal assertion is stated below. Multiplying (1.1) by a power of $Q$, we obtain the relations

$$
2 \pi \sum_{s} s Q^{l} \Phi_{k-r+s, r-s, s}-\sum_{s} Q^{l+1} \Phi_{k-r+s, r-s, s-2}=0
$$

for $l \in \mathbb{N}_{0}, r \in \mathbb{N}$, and $k \in\{0, \ldots, n+r-2\}$. We want to show that linear combinations of such relations yield all linear relations between basic tensor valuations. At one point of the proof, we need the existence of some convex body $K$ for which $\Phi_{k, 0, s}(K)$ with given $s \geq 2$ does not contain $Q$ as a factor. The somewhat intricate verification of this property is deferred to the Appendix (see Lemma 5.3), so that first the main line of the argument can be presented.

For the proof of the main assertion, it is sufficient to consider tensor valuations of given rank and degree of homogeneity. For $p, k \in \mathbb{N}_{0}$, let $T_{p, k}$ denote the real vector space of tensor valuations of rank $p$ and homogeneity degree $k$ that are continuous and isometry covariant. If we put

$$
\begin{aligned}
I_{p, k}:=\{(l, m, r, s): & l, r, s \in \mathbb{N}_{0}, m \in\{0, \ldots, n-1\} \\
& \text { or }(m, s)=(n, 0), 2 l+r+s=p, m+r=k\},
\end{aligned}
$$

then

$$
B_{p, k}:=\left\{Q^{l} \Phi_{m, r, s}:(l, m, r, s) \in I_{p, k}\right\}
$$

is the set of basic tensor valuations contained in $T_{p, k}$. Alesker's characterization theorem can be stated as

$$
T_{p, k}=\operatorname{lin} B_{p, k}
$$

In particular, $T_{p, k}=\{0\}$ for $k>n+p$.

For $k \leq n+p$, we define a $(p, k)$-dependence as a function $\alpha: I_{p, k} \rightarrow \mathbb{R}$ such that

$$
\sum_{(l, m, r, s) \in I_{p, k}} \alpha(l, m, r, s) Q^{l} \Phi_{m, r, s}=0 .
$$

The value $\alpha(l, m, r, s)$ will be called the $(l, m, r, s)$-coordinate of $\alpha$. With the natural definitions, the set of $(p, k)$-dependences is a real vector space, which we denote by $D_{p, k}$. Any equation (2.1) with $2 l+r=p$ defines a $(p, k)$-dependence $\alpha$, by taking for $\alpha\left(l^{\prime}, m^{\prime}, r^{\prime}, s^{\prime}\right)$ the coefficient of $Q^{l^{\prime}} \Phi_{m^{\prime}, r^{\prime}, s^{\prime}}$ if this basic tensor valuation appears in the relation, and 0 otherwise. A $(p, k)$-dependence obtained in this way will be called a basic $(p, k)$-dependence.

Theorem 2.1. Let $p, k \in \mathbb{N}_{0}$ and $k \leq n+p$. The vector space $D_{p, k}$ of $(p, k)$-dependences is spanned by the basic $(p, k)$-dependences.

Proof. First, let $p=0$. Then $k \in\{0, \ldots, n\}$ and, for any such $k, I_{0, k}=\{(0, k, 0,0)\}$, i.e., $B_{0, k}=\left\{\Phi_{k, 0,0}\right\}$. The map $(0, k, 0,0) \mapsto 0$ is a basic $(0, k)$-dependence which spans $D_{0, k}=\{0\}$, since $\Phi_{k, 0,0}$ is not the zero valuation.

Next, let $p=1$, and hence $k \in\{0, \ldots, n+1\}$. From (2.1) we get

$$
2 \pi \Phi_{m, 0,1}=0 \text { for } m=0, \ldots, n-1 \text {. }
$$

We have

$$
B_{1,0}=\left\{\Phi_{0,0,1}\right\}
$$


and $\Phi_{0,0,1}=0$; hence $D_{1,0}$ is one-dimensional and is spanned by the basic $(1,0)$-dependence given by $(2.2)$ for $m=0$.

We note that for $m \in\{0, \ldots, n\}$ we have $\Phi_{m, 1,0} \neq 0$, since $\Phi_{m, 1,0}(C) \neq 0$ for a cube $C$ having one of its vertices at the origin.

Let $k \in\{1, \ldots, n-1\}$. Then

$$
B_{1, k}=\left\{\Phi_{k, 0,1}, \Phi_{k-1,1,0}\right\},
$$

where $\Phi_{k, 0,1}=0$ by $(2.2)$ and $\Phi_{k-1,1,0} \neq 0$. Hence, $D_{1, k}$ is again one-dimensional and is spanned by the basic $(1, k)$-dependence.

Finally, let $k \in\{n, n+1\}$. Then

$$
B_{1, k}=\left\{\Phi_{k-1,1,0}\right\}
$$

and $\Phi_{k-1,1,0} \neq 0$, hence $D_{1, k}=\{0\}$. Thus, in each case, $D_{1, k}$ is spanned by the basic $(1, k)$-dependences.

Now let $p \geq 2$ and assume that the assertion of the theorem is true for the spaces $D_{r, k}$ with $r<p$ and arbitrary $k \leq n+r$. We shall show that then the assertion of the theorem is true for $D_{p, k}$ with $k \leq n+p$.

We start with $k=0$. We have

$$
B_{p, 0}=\left\{Q^{l} \Phi_{0,0, p-2 l}: l \in \mathbb{N}_{0}, 2 l \leq p\right\} .
$$

From (2.1), we obtain (with $\Phi_{0,0,-1}:=0$ )

$$
2 \pi r Q^{(p-r) / 2} \Phi_{0,0, r}-Q^{1+(p-r) / 2} \Phi_{0,0, r-2}=0
$$

for $r=1,3, \ldots, p$ if $p$ is odd, and $r=2,4, \ldots, p$ if $p$ is even.

Relations (2.3) define the corresponding basic $(p, 0)$-dependences. Using (2.3) repeatedly, we obtain

$$
T_{p, 0}=\{0\} \text { for odd } p, \quad T_{p, 0}=\operatorname{lin}\left\{Q^{p / 2} \Phi_{0,0,0}\right\} \neq\{0\} \text { for even } p .
$$

This shows in each case that the space spanned by the basic $(p, 0)$-dependences and the space $D_{p, 0}$ of all $(p, 0)$-dependences have the same dimension.

Now we assume that $k \geq 1$. If $k \in\{n+p-1, n+p\}$, then $I_{p, k}=\{(0, k-p, p, 0)\}$ and $\Phi_{k-p, p, 0} \neq 0$. In these two cases, $D_{p, k-p}=\{0\}$ is spanned by the basic $(p, k-p)$ dependence $(0, k-p, p, 0) \mapsto 0$. Hence, throughout the following, we shall assume that $1 \leq k \leq n+p-2$.

Let $\alpha \in D_{p, k}$, so that

$$
\sum_{(l, m, r, s) \in I_{p, k}} \alpha(l, m, r, s) Q^{l} \Phi_{m, r, s}=0 .
$$

Since here $2 l+r+s=p$ and $m+r=k$, we rewrite the relation as

$$
\sum_{l, s \in \mathbb{N}_{0}} \alpha_{l, s} Q^{l} \Phi_{k-p+s+2 l, p-s-2 l, s}=0
$$

with $\alpha_{l, s}:=\alpha(l, k-p+s+2 l, p-s-2 l, s)$ (setting $\alpha_{l, s}:=0$ in the undefined cases).

Assume, first, that $\alpha_{0, s} \Phi_{k-p+s, p-s, s}=0$ for $s=0, \ldots, p$. Then (2.5) gives

$$
Q \sum_{l \geq 1, s \geq 0} \alpha_{l, s} Q^{l-1} \Phi_{k-p+s+2 l, p-s-2 l, s}=0 .
$$

Since $\mathbb{T}$ has no zero divisors, this implies the linear relation

$$
\sum_{l \geq 1, s \geq 0} \alpha_{l, s} Q^{l-1} \Phi_{k-p+s+2 l, p-s-2 l, s}=0
$$


between basic tensor valuations of rank $p-2$ and homogeneity degree $k$ and hence determines a $(p-2, k)$-dependence. By the inductive assumption, $D_{p-2, k}$ is spanned by the basic $(p-2, k)$-dependences. But then the $(p, k)$-dependence corresponding to $(2.5)$ is spanned by basic $(p, k)$-dependences (we use the linear map $D_{p-2, k} \rightarrow D_{p, k}$ that is induced by multiplying linear relations by $Q)$.

We may assume that either $\alpha_{0, s} \Phi_{k-p+s, p-s, s} \neq 0$ for some $s \in\{0, \ldots, p-1\}$, or $\alpha_{0, s} \Phi_{k-p+s, p-s, s}=0$ for all $s \in\{0, \ldots, p-1\}$ and $\alpha_{0, p} \Phi_{k, 0, p} \neq 0$.

To analyze the coefficients in (2.5), we use the translation covariance of the basic tensor valuations. For $K \in \mathcal{K}^{n}$ and $t \in \mathbb{R}^{n}$, we have

$$
\Phi_{k^{\prime}, r^{\prime}, s^{\prime}}(K+t)=\sum_{j=0}^{r^{\prime}} \frac{1}{j !} \Phi_{k^{\prime}, r^{\prime}-j, s^{\prime}}(K) t^{j} .
$$

Applying (2.6) to (2.5) and comparing the coefficients of $t^{1}$, we obtain

$$
\sum_{l, s \in \mathbb{N}_{0}} \alpha_{l, s} Q^{l} \Phi_{k-p+s+2 l, p-s-2 l-1, s}=0 .
$$

This is a linear relation between basic tensor valuations of rank $p-1$ and homogeneity degree $k-1$ and hence determines a $(p-1, k-1)$-dependence $\bar{\alpha} \in D_{p-1, k-1}$. By the inductive assumption, $D_{p-1, k-1}$ is spanned by the basic $(p-1, k-1)$-dependences. From this, we shall derive information about the coefficients $\alpha_{l, s}$ in (2.5).

First we show that $\alpha_{0,0} \Phi_{k-p, p, 0}=0$. To see this, assume that $\alpha_{0,0} \Phi_{k-p, p, 0} \neq 0$. Hence $0 \leq k-p \leq n$ and $\alpha_{0,0} \Phi_{k-p, p-1,0}$ is a nonvanishing summand in (2.7). The relations (2.1) do not involve a nonzero multiple of the functional $\Phi_{k-p, p-1,0}$; hence any basic $(p-1, k-1)$-dependence has $(0, k-p, p-1,0)$-coordinate zero. By the inductive assumption, the same is true for every element of $D_{p-1, k-1}$. This is a contradiction.

Hence, we know that $\alpha_{0, s_{0}} \Phi_{k-p+s_{0}, p-s_{0}, s_{0}} \neq 0$ for some $s_{0} \in\{1, \ldots, p\}$. Assume first that $s_{0} \in\{1, \ldots, p-1\}$. This implies that $0 \leq k-p+s_{0} \leq n-1$; thus (2.7) involves the nonvanishing summand $\alpha_{0, s_{0}} \Phi_{k-p+s_{0}, p-s_{0}-1, s_{0}}$. The only relation from (2.1) that involves $\Phi_{k-p+s_{0}, p-s_{0}-1, s_{0}}$ is

$$
2 \pi \sum_{s=1}^{p-1} s \Phi_{k-p+s, p-s-1, s}-Q \sum_{s=2}^{p-1} \Phi_{k-p+s, p-s-1, s-2}=0 .
$$

This is also the uniquely determined linear relation of the form (2.1) which involves one of the functionals $\Phi_{k-p+1, p-2,1}, \ldots, \Phi_{k-1,0, p-1}$. It follows that in any basic $(p-1, k-1)$ dependence the $(p-1)$-tuple of $(0, k-p+s, p-s-1, s)$-coordinates, $s=1, \ldots, p-1$, is of the form $c \cdot 2 \pi(1, \ldots, p-1)$ with a constant $c$. By the inductive assumption, the same is true for every element of $D_{p-1, k-1}$, in particular for $\bar{\alpha}$. We conclude that $\alpha_{0, s}=c \cdot 2 \pi s$ for $s=1, \ldots, p-1$, whence

$$
\alpha_{0, s}=\frac{\alpha_{0, s_{0}}}{s_{0}} s, \quad s=1, \ldots, p-1
$$

Thus, (2.5) is equivalent to the relation

$$
2 \pi \alpha \sum_{s=1}^{p-1} s \Phi_{k-p+s, p-s, s}+\alpha_{0, p} \Phi_{k, 0, p}+\sum_{l \geq 1, s \geq 0} \alpha_{l, s} Q^{l} \Phi_{k-p+s+2 l, p-s-2 l, s}=0
$$

with $\alpha=\alpha_{0, s_{0}} /\left(2 \pi s_{0}\right) \neq 0$. In the still excluded case $s_{0}=p$, relation (2.8) is also fulfilled, because then we can assume $\alpha_{0, s}=0$ for $s=1, \ldots, p-1$. In this case, (2.8) is true with $\alpha=0$ and $\alpha_{0, p} \neq 0$. 
Subtracting a multiple of (2.1) (with $l=0, r=p$ ) from (2.8), we arrive at

$$
\left(\alpha_{0, p}-2 \pi \alpha p\right) \Phi_{k, 0, p}-\sum_{l \geq 1, s \geq 0} \tilde{\alpha}_{l, s} Q^{l} \Phi_{k-p+s+2 l, p-s-2 l, s}=0
$$

with suitable constants $\tilde{\alpha}_{l, s} \in \mathbb{R}$. This shows that for each $K \in \mathcal{K}^{n}$ there is some $v \in \mathbb{T}^{p-2}$ such that

$$
\left(\alpha_{0, p}-2 \pi \alpha p\right) \Phi_{k, 0, p}(K)=Q v .
$$

If $1 \leq k \leq n-1$, then there exists a convex body $K$ for which $\Phi_{k, 0, p}(K)$ cannot be written in the form $Q v$ with $v \in \mathbb{T}^{p-2}$. The construction of such a body is postponed to the Appendix (see Lemma 5.3). Taking its existence for granted, we deduce that $\alpha_{0, p}=2 \pi \alpha p \neq 0$, so that (2.5) takes the equivalent form

$$
2 \pi \alpha \sum_{s=1}^{p} s \Phi_{k-p+s, p-s, s}+\sum_{l \geq 1, s \geq 0} \alpha_{l, s} Q^{l} \Phi_{k-p+s+2 l, p-s-2 l, s}=0 .
$$

Subtracting again a multiple of $(2.1)$ (with $l=0, r=p$ ) from this equation, we obtain

$$
\alpha Q \sum_{s} \Phi_{k-p+s, p-s, s-2}+\sum_{l \geq 1, s \geq 0} \alpha_{l, s} Q^{l} \Phi_{k-p+s+2 l, p-s-2 l, s}=0,
$$

which is equivalent to

$$
Q \sum_{l \geq 1, s \geq 0} \beta_{l, s} Q^{l-1} \Phi_{k-p+s+2 l, p-s-2 l, s}=0,
$$

with suitable constants $\beta_{l, s} \in \mathbb{R}$. The latter is equivalent to

$$
\sum_{l \geq 1, s \geq 0} \beta_{l, s} Q^{l-1} \Phi_{k-p+s+2 l, p-s-2 l, s}=0 .
$$

Relation (2.10) defines a $(p-2, k)$-dependence. By the inductive assumption, it is spanned by basic $(p-2, k)$-dependences. But then $(2.9)$, which is equivalent to $(2.5)$, shows that the $(p, k)$-dependence corresponding to $(2.5)$ is spanned by basic $(p, k)$-dependences.

If $k \geq n$, then $\Phi_{k, 0, p}$ is equal to zero by definition, and we can argue as before.

This shows that in any case the assertion of the theorem is true for functionals of rank $p$, which completes the induction.

Remark 2.2. Let $p \geq 2$. Theorem 2.1 implies that any linear relation between functionals from $B_{p, k}$, involving a nonzero multiple of one of the nontrivial functionals $\Phi_{k-p+1, p-1,1}, \ldots, \Phi_{k, 0, p}$, necessarily is, up to a factor, of the form

$$
2 \pi \sum_{s} s \Phi_{k-p+s, p-s, s}=Q \Phi
$$

with some $\Phi \in T_{p-2, k}$. Moreover, there is no such linear relation that involves a nonzero multiple of $\Phi_{k-p, p, 0}$ as a summand whenever $k \in\{p, \ldots, n+p\}$. Both facts follow since

$$
2 \pi \sum_{s} s \Phi_{k-p+s, p-s, s}=Q \sum_{s} \Phi_{k-p+s, p-s, s-2}
$$

is unique among the relations $(2.1)$ in that it involves one of $\Phi_{k-p+1, p-1,1}, \ldots, \Phi_{k, 0, p}$ as a summand, and none of the relations (2.1) involve $\Phi_{k-p, p, 0}$ with a nonzero real factor.

As a consequence, we obtain the following corollary. The assumptions $p \geq 2$ and $k \geq 1$ in the corollary cannot be avoided.

Corollary 2.3. Let $p \geq 2$ and $k \geq 1$ with $k \leq n+p$. Then the functionals $\Phi_{k-p+s, p-s, s}$ with $(0, k-p+s, p-s, s) \in I_{p, k}$ are linearly independent. 
Proof. For $k=n+p$ the only functional occurring is $\Phi_{n, p, 0} \neq 0$, and for $k=n+p-1$ the only one is $\Phi_{n-1, p, 0} \neq 0$. Hence, in the following we can assume that $k \leq n+p-2$.

For $p=2$ and $k \leq n$, we have to consider the functionals $\Phi_{k-2+s, 2-s, s}$ for $s \in\{0,1,2\}$, which are nontrivial. Suppose there were a linear relation between them. By Remark 2.2 , it must (up to a nonzero constant) be given by

$$
2 \pi \sum_{s} s \Phi_{k-2+s, 2-s, s}=0 .
$$

From (2.1) we deduce then that

$$
\sum_{s} \Phi_{k-2+s, 2-s, s-2}=0
$$

which reduces to $\Phi_{k, 0,0}=0$. Since $k \leq n$, this yields a contradiction.

For $p=3$ and $k \leq n+1$, we argue similarly and arrive at the linear relation

$$
2 \pi \sum_{s} s \Phi_{k-3+s, 3-s, s}=0 .
$$

Using again (2.1), we deduce that $\Phi_{k-1,1,0}=0$. Here we use the fact that always $\Phi_{k, 0,1}=0$. Since $0 \leq k-1 \leq n$, this is a contradiction.

Now let $p \geq 4, k \geq 1$, and $k \leq n+p-2$. Assume that the assertion of the corollary is proved in $B_{r, k}$ for $2 \leq r<p$ and $k \geq 1$ with $k \leq n+r$. Assume there is a linear relation between the Minkowski tensors in question. By Remark 2.2, it is (up to a nonzero constant) given by

$$
2 \pi \sum_{s} s \Phi_{k-p+s, p-s, s}=0
$$

By (2.1), we conclude that

$$
\sum_{s} \Phi_{k-p+s, p-s, s-2}=0
$$

If $k-p+2 \geq 0$, then (2.11) involves $\Phi_{k-p+2, p-2,0}$ with $p-2 \geq 2$ and $0 \leq k-p+2 \leq n$, a contradiction to Remark (2.2) (or to the inductive assumption). If $k-p+2 \leq-1$, i.e., $p-k-2 \geq 1$, then (2.11) involves $\Phi_{0, k, p-k-2} \in B_{p-2, k}$ as a nontrivial summand, where $p-2 \geq 2$ and $k \geq 1$. This contradicts the inductive assumption and thus completes the argument.

Corollary 2.3 and the preceding remark will play a crucial role in the arguments of the next section.

\section{$\S 3$. Dimension AND BASIS}

In this section, we use Theorem 2.1 for determining the dimension of the vector space

$$
T_{p, k}=\operatorname{lin}\left\{Q^{l} \Phi_{m, r, s}:(l, m, r, s) \in I_{p, k}\right\}
$$

of $\mathbb{T}^{p}$-valued, continuous, isometry covariant valuations on $\mathcal{K}^{n}$, which are homogeneous of degree $k$. In the course of the proof (see Lemma 3.2), we also determine a particular basis for this vector space. Examples will be given in the subsequent section.

Theorem 3.1. Let $p \in \mathbb{N}_{0}$, and let $0 \leq k \leq n+p$. Put

$$
j_{0}:=\min \left\{\left\lfloor\frac{n+p-k}{2}\right\rfloor,\left\lfloor\frac{p}{2}\right\rfloor\right\} \quad \text { and } \quad j_{1}:=\max \left\{-1,\left\lfloor\frac{p-k}{2}\right\rfloor\right\} .
$$

Then

$$
\operatorname{dim} T_{p, k}=j_{0}\left(\min \{1, n-k\}+p-j_{0}\right)+1-\left(j_{1}+1\right)\left(p-k-j_{1}\right) .
$$


Proof. First, we consider the case of $p=0$. For $0 \leq k \leq n$,

$$
T_{0, k}=\operatorname{lin}\left\{\Phi_{k, 0,0}\right\}
$$

and $\Phi_{k, 0,0} \neq 0$, hence

$$
\operatorname{dim} T_{0, k}=1
$$

This is consistent with (3.1).

For $p=1$, we know from $\S 2$ that $T_{1,0}=\{0\}$ and, for $k \in\{1, \ldots, n+1\}$,

$$
T_{1, k}=\operatorname{lin}\left\{\Phi_{k-1,1,0}\right\} \neq\{0\},
$$

whence

$$
\operatorname{dim} T_{1, k}= \begin{cases}0 & \text { if } k=0 \\ 1 & \text { if } k \in\{1, \ldots, n+1\} .\end{cases}
$$

This, too, is consistent with (3.1).

Let $p \geq 2$. The case $k=0$ is easy. By (2.4),

$$
\operatorname{dim} T_{p, 0}= \begin{cases}0 & \text { if } p \text { is odd } \\ 1 & \text { if } p \text { is even }\end{cases}
$$

which is consistent with (3.1).

From now on we assume that $k \geq 1$. We shall decompose $T_{p, k}$ into a sum of linear subspaces and then into a direct sum. For this purpose, for $j \in \mathbb{N}_{0}$ with $0 \leq j \leq j_{0}$ we define the linear subspaces

$$
T_{p, k}^{j}:=\operatorname{lin}\left\{Q^{j} \Phi_{k-p+2 j+s, p-2 j-s, s}: 0 \leq s \leq p-2 j\right\},
$$

and put $T_{p, k}^{j}:=\{0\}$ for all other integers $j$. Then we have

$$
\sum_{j} T_{p, k}^{j}=T_{p, k}
$$

Here summation effectively extends over $j \in\left\{0, \ldots, j_{0}\right\}$.

First we determine the dimension of $T_{p, k}^{j}$ for $0 \leq j \leq j_{0}$. Let $A_{p, k}^{j}$ denote the number of basic tensor valuations $Q^{j} \Phi_{k-p+2 j+s, p-2 j-s, s} \in T_{p, k}^{j}$ with $s \in\{0, \ldots, p-2 j\}$. In order to ensure that $Q^{j} \Phi_{k-p+2 j+s, p-2 j-s, s}$ be a basic tensor valuation, we must have either

$$
0 \leq k-p+2 j+s \leq n-1 \text { and } 0 \leq s \leq p-2 j
$$

(observe that by the definition of $j_{0}$ we always have $p-2 j \geq 0$ ), or

$$
k-p+2 j+s=n \text { and } s=0,
$$

and each of these conditions is also sufficient. The first set of conditions is equivalent to

$$
\max \{0, p-k-2 j\} \leq s \leq \min \{p-2 j, n+p-k-2 j-1\} .
$$

Note that this cannot be satisfied for $2 j=n+p-k$. The second set of conditions is equivalent to

$$
2 j=n+p-k \text { and } s=0 .
$$

Hence, we obtain

$$
A_{p, k}^{j}=\min \{p-2 j+1, n+p-k-2 j\}-\max \{0, p-k-2 j\}+\delta_{2 j, n+p-k},
$$

where $\delta$ denotes the Kronecker symbol. Equation (3.4) is true for $0 \leq j \leq j_{0}$. In all other cases we have $A_{p, k}^{j}=0$.

Let $j$ be fixed. Since $\mathbb{T}$ has no zero divisors, a sequence of functionals of the form $Q^{j} \Phi_{k-p+2 j+s, p-2 j-s, s} \in T_{p, k}^{j}$ is linearly independent if and only if the corresponding 
sequence of functionals $\Phi_{k-p+2 j+s, p-2 j-s, s} \in T_{p-2 j, k}^{0}$ is linearly independent. Thus, we get

$$
\operatorname{dim} T_{p, k}^{j}=\operatorname{dim} T_{p-2 j, k}^{0} .
$$

Since $k \geq 1$ and $A_{p, k}^{j}=A_{p-2 j, k}^{0}$, Corollary 2.3 implies that

$$
\operatorname{dim} T_{p, k}^{j}=\operatorname{dim} T_{p-2 j, k}^{0}=A_{p-2 j, k}^{0}=A_{p, k}^{j}
$$

for all $j \in \mathbb{N}_{0}$ with $j \leq j_{0}$ and $j \leq(p / 2)-1$; here the second restriction is imposed in view of the assumption $p-2 j \geq 2$ needed in the application of Corollary 2.3. The remaining cases are easily settled. If $p$ is odd, we may have $p-2 j_{0}=1$. Then (3.3) yields

$$
\operatorname{dim} T_{p, k}^{j_{0}}=\operatorname{dim} T_{1, k}^{0}=\operatorname{dim} T_{1, k}=1,
$$

since $k \geq 1$. If $p$ is even, we may have $p-2 j_{0}=0$. Then (3.2) shows that

$$
\operatorname{dim} T_{p, k}^{j_{0}}=\operatorname{dim} T_{0, k}^{0}=\operatorname{dim} T_{0, k}=1 .
$$

For $j_{0}=0$, we have $T_{p, k}=T_{p, k}^{0}$. The dimension $\operatorname{dim} T_{p, k}^{0}$ has already been determined in (3.5), (3.6), and (3.7). Since $p \geq 2$, the definition of $j_{0}$ implies that $n+p-k=0$ or $n+p-k=1$. Hence, (3.4) and (3.5) imply

$$
\begin{aligned}
\operatorname{dim} T_{p, k}^{0} & =A_{p, k}^{0}=\min \{p+1, n+p-k\}-\max \{0, p-k\}+\delta_{0, n+p-k} \\
& =(n+p-k)-0+\delta_{0, n+p-k}=1,
\end{aligned}
$$

which is consistent with (3.1).

Now we assume that $j_{0} \geq 1$ and transform the right-hand side of

$$
T_{p, k}=\sum_{j} T_{p, k}^{j}
$$

into a direct sum. For each of the corresponding summands $\bar{T}_{p, k}^{j}$, a basis is provided by the basic tensor valuations in (3.8). A counting argument then completes the proof.

Lemma 3.2. Let $p \geq 2, k \geq 1$ be given. Let $j_{0} \geq 1$. For $0 \leq j<j_{0}$, put $s_{j}:=$ $\max \{1, p-k-2 j\} \in\{1, \ldots, p-2 j-1\}$. Then $\Phi_{k-p+2 j+s_{j}, p-2 j-s_{j}, s_{j}}$ is nontrivial. Define

$$
\bar{T}_{p, k}^{j}:=\operatorname{lin}\left\{Q^{j} \Phi_{k-p+2 j+s, p-2 j-s, s}: s \in\{0, \ldots, p-2 j\} \backslash\left\{s_{j}\right\}\right\} .
$$

Then

$$
\operatorname{dim} \bar{T}_{p, k}^{j}=\operatorname{dim} T_{p, k}^{j}-1
$$

and

$$
T_{p, k}=T_{p, k}^{0}+\ldots+T_{p, k}^{j_{0}}=\bar{T}_{p, k}^{0} \oplus \ldots \oplus \bar{T}_{p, k}^{j_{0}-1} \oplus T_{p, k}^{j_{0}} .
$$

Proof. Let $0 \leq j<j_{0}$. By the definition of $j_{0}$, we have $n+p-k \geq 2 j_{0}$, whence $k-p+2 j \leq n-2$. Put $s_{j}:=\max \{1, p-k-2 j\}$. Then $\Phi_{k-p+2 j+s_{j}, p-2 j-s_{j}, s_{j}}$ is equal either to $\Phi_{k-p+2 j+1, p-2 j-1,1}$ with $1 \leq k-p+2 j+1 \leq n-1$, or to $\Phi_{0, k, p-k-2 j}$ with $p-k-2 j>1$. In both cases, this is a nontrivial functional. By Corollary 2.3, the basic tensor valuations spanning $T_{p, k}^{j}$ are linearly independent. Hence, if one of these is deleted, then the dimension of the span is reduced by one. This proves (3.9).

We prove (3.10) by induction. For this, we assert that the relation

$$
T_{p, k}^{0}+\ldots+T_{p, k}^{j}=\bar{T}_{p, k}^{0} \oplus \ldots \oplus \bar{T}_{p, k}^{j-1} \oplus T_{p, k}^{j}
$$

is true for $1 \leq j \leq j_{0}$. This will be proved by induction on $j$. The assertion of the lemma then follows by putting $j=j_{0}$. 
First, let $j=1 \leq j_{0}$. If $\Phi \in T_{p, k}^{0} \cap T_{p, k}^{1}$, then

$$
\Phi=\sum_{s} \alpha_{s} \Phi_{k-p+s, p-s, s}=\sum_{s} \beta_{s} Q \Phi_{k-p+s, p-s, s-2}
$$

with suitable constants $\alpha_{s}, \beta_{s} \in \mathbb{R}$. By Theorem 2.1, the linear relation

$$
\sum_{s} \alpha_{s} \Phi_{k-p+s, p-s, s}-\sum_{s} \beta_{s} Q \Phi_{k-p+s, p-s, s-2}=0
$$

is obtained from the linear relation

$$
2 \pi \sum_{s} s \Phi_{k-p+s, p-s, s}-\sum_{s} Q \Phi_{k-p+s, p-s, s-2}=0
$$

by multiplication by a real constant. We conclude that

$$
T_{p, k}^{0} \cap T_{p, k}^{1}=\operatorname{lin}\left\{\sum_{s} s \Phi_{k-p+s, p-s, s}\right\} .
$$

Since $s_{0} \Phi_{k-p+s_{0}, p-s_{0}, s_{0}}$ is nontrivial, and by Corollary 2.3, we obtain

$$
\operatorname{dim} T_{p, k}^{0} \cap T_{p, k}^{1}=1 .
$$

By the definition of $\bar{T}_{p, k}^{0}$, we have

$$
\bar{T}_{p, k}^{0} \cap T_{p, k}^{1}=\{0\}
$$

thus

$$
T_{p, k}^{0}+T_{p, k}^{1}=\bar{T}_{p, k}^{0} \oplus T_{p, k}^{1} .
$$

If $j_{0}=1$, this already completes the proof.

Let $2 \leq j \leq j_{0}$ and assume that (3.11) has been proved for all integers less than $j$. By the inductive assumption,

$$
T_{p, k}^{0}+\ldots+T_{p, k}^{j}=\bar{T}_{p, k}^{0} \oplus \ldots \oplus \bar{T}_{p, k}^{j-2} \oplus T_{p, k}^{j-1}+T_{p, k}^{j} .
$$

Every functional

satisfies

$$
\Phi \in\left(\bar{T}_{p, k}^{0} \oplus \ldots \oplus \bar{T}_{p, k}^{j-2} \oplus T_{p, k}^{j-1}\right) \cap T_{p, k}^{j}
$$

$$
\begin{aligned}
\Phi & =\sum_{j=0}^{j-2} \sum_{s \neq s_{j}} \alpha_{s}^{(j)} Q^{j} \Phi_{k-p+2 j+s, p-2 j-s, s}+\sum_{s} \beta_{s} Q^{j-1} \Phi_{k-p+2(j-1)+s, p-2(j-1)-s, s} \\
& =\sum_{s} \gamma_{s} Q^{j} \Phi_{k-p+2 j+s, p-2 j-s, s}
\end{aligned}
$$

with suitably chosen $\alpha_{s}^{(0)}, \ldots, \alpha_{s}^{(j-2)}, \beta_{s}, \gamma_{s} \in \mathbb{R}$. Since $\Phi_{k-p+s_{0}, p-s_{0}, s_{0}}$ is missing in the first inner sum (corresponding to $j=0$ ), Theorem 2.1 and Remark 2.2 imply that $\alpha_{s}^{(0)}=0$ for all $s$. Then we can conclude similarly that $\alpha_{s}^{(1)}=0$ for all $s$, and so on until $\alpha_{s}^{(j-2)}=0$ for all $s$. Hence, we get

$$
\sum_{s} \beta_{s} \Phi_{k-p+2(j-1)+s, p-2(j-1)-s, s}=\sum_{s} \gamma_{s} Q \Phi_{k-p+2 j+s, p-2 j-s, s}
$$

As above, we can infer that

$$
\left(\bar{T}_{p, k}^{0} \oplus \ldots \oplus \bar{T}_{p, k}^{j-2} \oplus T_{p, k}^{j-1}\right) \cap T_{p, k}^{j}=\operatorname{lin}\left\{Q^{j-1} \sum_{s} s \Phi_{k-p+2(j-1)+s, p-2(j-1)-s, s}\right\} .
$$

It follows as before that the space on the right-hand side has dimension one and that

$$
\left(\bar{T}_{p, k}^{0} \oplus \ldots \oplus \bar{T}_{p, k}^{j-2} \oplus \bar{T}_{p, k}^{j-1}\right) \cap T_{p, k}^{j}=\{0\},
$$


and thus

$$
T_{p, k}^{0}+\ldots+T_{p, k}^{j}=\bar{T}_{p, k}^{0} \oplus \ldots \oplus \bar{T}_{p, k}^{j-1} \oplus T_{p, k}^{j} .
$$

This finishes the induction and, with it, the proof of the lemma.

We have obtained the decomposition

$$
T_{p, k}=\left(\bigoplus_{j=0}^{j_{0}-1} \bar{T}_{p, k}^{j}\right) \oplus T_{p, k}^{j_{0}}
$$

and for the corresponding dimensions we get

$$
\operatorname{dim} T_{p, k}=\sum_{j=0}^{j_{0}-1}\left(\operatorname{dim} T_{p, k}^{j}-1\right)+\operatorname{dim} T_{p, k}^{j_{0}} .
$$

Equation (3.5) shows that $\operatorname{dim} T_{p, k}^{j}=A_{p, k}^{j}$ for $j=0, \ldots, j_{0}-1$. From (3.5) and (3.4) in the case where $j_{0} \leq(p / 2)-1$, and from (3.6) or (3.7) in the case where $j_{0}>(p / 2)-1$, we deduce that

$$
\operatorname{dim} T_{p, k}^{j_{0}}=1
$$

Hence, (3.12) implies

$$
\operatorname{dim} T_{p, k}=\sum_{j=0}^{j_{0}-1} A_{p, k}^{j}-j_{0}+1
$$

Using (3.4), we finally get

$$
\begin{aligned}
\operatorname{dim} T_{p, k} & =\sum_{j=0}^{j_{0}-1}(\min \{p-2 j+1, n+p-k-2 j\}-\max \{0, p-k-2 j\})-j_{0}+1 \\
& =\sum_{j=0}^{j_{0}-1}(\min \{1, n-k\}+p-2 j)-\sum_{j=0}^{j_{0}-1} \max \{0, p-k-2 j\}-j_{0}+1 \\
& =j_{0}(\min \{1, n-k\}+p)-j_{0}\left(j_{0}-1\right)-j_{0}+1-\sum_{j=0}^{j_{0}-1} \max \{0, p-k-2 j\} \\
& =j_{0}\left(\min \{1, n-k\}+p-j_{0}\right)+1-\sum_{j=0}^{j_{0}-1} \max \{0, p-k-2 j\}
\end{aligned}
$$

Since $p-k-2 j \geq 0$ if and only if $2 j \leq p-k$, we can simplify the preceding expression by putting

$$
j_{1}:=\max \left\{-1,\left\lfloor\frac{p-k}{2}\right\rfloor\right\}
$$

and writing

$$
\begin{aligned}
\operatorname{dim} T_{p, k} & =j_{0}\left(\min \{1, n-k\}+p-j_{0}\right)+1-\sum_{j=0}^{\max \left\{0, j_{1}\right\}}(p-k-2 j) \\
& =j_{0}\left(\min \{1, n-k\}+p-j_{0}\right)+1-\left(j_{1}+1\right)\left(p-k-j_{1}\right) .
\end{aligned}
$$

Here we have used the fact that either $j_{1} \leq j_{0}-1$, or $j_{1}=j_{0}$ and $p-k-2 j_{0}=0$. This confirms the asserted expression for the dimension. 


\section{$\S 4$. BASES FOR SMALL RANKS}

Theorem 3.1 provides the dimension of $T_{p, k}$; the proof shows how a basis of $T_{p, k}$ can be found in principle. Now we apply the employed method to determine explicit bases for the spaces $T_{p, k}$ of ranks $p \leq 3$.

Proposition. The following list provides bases for the spaces $T_{p, k}$ up to rank three. $\underline{\text { Rank } 0}$

- $T_{0, k}$ for $k=0, \ldots, n$ : a basis is $\left\{\Phi_{k, 0,0}\right\}$.

\section{$\underline{\operatorname{Rank} 1}$}

- $T_{1,0}=\{0\}$.

- $T_{0, k}$ for $k=1, \ldots, n+1$ : a basis is $\left\{\Phi_{k-1,1,0}\right\}$.

\section{$\underline{\operatorname{Rank} 2}$}

- $T_{2,0}:$ a basis is $\left\{Q \Phi_{0,0,0}\right\}$.

- $T_{2,1}$ : a basis is $\left\{\Phi_{1,0,2}, Q \Phi_{1,0,0}\right\}$.

- $T_{2, k}$ for $k=2, \ldots, n-1$ : a basis is $\left\{\Phi_{k, 0,2}, \Phi_{k-2,2,0}, Q \Phi_{k, 0,0}\right\}$.

- $T_{2, n}:$ a basis is $\left\{\Phi_{n-2,2,0}, Q \Phi_{n, 0,0}\right\}$.

- $T_{2, k}$ for $k=n+1, n+2$ : a basis is $\left\{\Phi_{k-2,2,0}\right\}$.

\section{$\underline{\operatorname{Rank} 3}$}

- $T_{3,0}$ : a basis is $\left\{\Phi_{0,0,3}\right\}$.

- $T_{3,1}$ : a basis is $\left\{\Phi_{1,0,3}, Q \Phi_{0,1,0}\right\}$.

- $T_{3, k}$ for $k=2, \ldots, n+1$ : a basis is $\left\{\Phi_{k, 0,3}, \Phi_{k-1,1,2}, \Phi_{k-3,3,0}, Q \Phi_{k-1,1,0}\right\}$, with trivial functionals deleted.

- $T_{3, k}$ for $k=n+2, n+3$ : a basis is $\left\{\Phi_{k-3,3,0}\right\}$.

Proof. For tensors of rank $p=0$ and $p=1$, bases are well known. Here we have

$$
T_{0, k}=\operatorname{lin}\left\{\Phi_{k, 0,0}\right\}, \quad 0 \leq k \leq n, \quad T_{1, k}=\operatorname{lin}\left\{\Phi_{k-1,1,0}\right\}, \quad 1 \leq k \leq n+1 .
$$

In addition, we remark that the tensor valuations $\Phi_{0,0,0}, \ldots, \Phi_{n, 0,0}$ are linearly independent, and $\Phi_{0,1,0}, \ldots, \Phi_{n, 1,0}$ are linearly independent.

Next we consider the case of $p=2$. For arbitrary $k \in \mathbb{N}_{0}$, we get

$$
T_{2, k}^{0}=\operatorname{lin}\left\{\Phi_{k, 0,2}, \Phi_{k-1,1,1}, \Phi_{k-2,2,0}\right\}, \quad T_{2, k}^{1}=\operatorname{lin}\left\{Q \Phi_{k, 0,0}\right\} .
$$

The linear relation (2.1) that corresponds to integers $k \in \mathbb{N}_{0}$ and $p=2$ is

$$
2 \pi \Phi_{k-1,1,1}+4 \pi \Phi_{k, 0,2}=Q \Phi_{k, 0,0}
$$

First, let $k=0$. Then $j_{0}=1, j_{1}=1$, and hence $\operatorname{dim} T_{2,0}=1$ by Theorem 3.1. Specifically, we have

$$
T_{2,0}^{0}=\operatorname{lin}\left\{\Phi_{0,0,2}\right\} \quad \text { and } \quad T_{2,0}^{1}=\operatorname{lin}\left\{Q \Phi_{0,0,0}\right\} .
$$

Equation (4.1) reduces to

$$
4 \pi \Phi_{0,0,2}=Q \Phi_{0,0,0},
$$

hence a basis of $T_{2,0}$ is precisely $\left\{Q \Phi_{0,0,0}\right\}$.

Now we consider the cases of $1 \leq k \leq n-1$, where $j_{0}=1$. For $k=1,2$ we obtain $j_{1}=0$, and otherwise we get $j_{1}=-1$. Hence, Theorem 3.1 yields $\operatorname{dim} T_{2, k}=2$ if $k=1$ and $\operatorname{dim} T_{2, k}=3$ otherwise.

The subspaces $T_{2, k}^{0}$ and $T_{2, k}^{1}$ have the same form as in the general case. In the present case, the functionals in (4.1) are nontrivial. Thus, we get

$$
\bar{T}_{2, k}^{0}=\operatorname{lin}\left\{\Phi_{k, 0,2}, \Phi_{k-2,2,0}\right\} .
$$


Therefore, a basis of $T_{2, k}=\bar{T}_{2, k}^{0} \oplus T_{2, k}^{1}$ is given by

$$
\left\{\Phi_{k, 0,2}, \Phi_{k-2,2,0}, Q \Phi_{k, 0,0}\right\},
$$

where $\Phi_{k-2,2,0}$ is deleted if $k=1$.

For $k=n$, we have $j_{0}=1$ and $j_{1}=0$ if $n=1,2$, but $j_{1}=-1$ in all other cases. Hence, Theorem 3.1 implies that $\operatorname{dim} T_{2, n}=1$ if $n=1$, and $\operatorname{dim} T_{2, n}=2$ if $n \geq 2$. We have

$$
T_{2, n}^{0}=\operatorname{lin}\left\{\Phi_{n-1,1,1}, \Phi_{n-2,2,0}\right\}
$$

and

$$
T_{2, n}^{1}=\operatorname{lin}\left\{Q \Phi_{n, 0,0}\right\} .
$$

The linear relation (4.1) shows that

$$
2 \pi \Phi_{n-1,1,1}=Q \Phi_{n, 0,0} .
$$

Therefore, we get

$$
\bar{T}_{2, n}^{0}=\operatorname{lin}\left\{\Phi_{n-2,2,0}\right\} .
$$

Hence, a basis of $T_{2, n}$ is $\left\{\Phi_{n-2,2,0}, Q \Phi_{n, 0,0}\right\}$, where the first functional must be deleted if $n=1$.

It remains to consider the cases of $n+1 \leq k \leq n+2$, where $j_{0}=0$ and $j_{1}=0$ if $n=1$ and $k=2$, and $j_{1}=-1$ in all other cases. In any case, Theorem 3.1 implies that $\operatorname{dim} T_{2, k}=1$. Moreover, we have

$$
T_{2, k}^{0}=\operatorname{lin}\left\{\Phi_{k-2,2,0}\right\}
$$

This finally leads to

$$
T_{2, k}=T_{2, k}^{0}=\operatorname{lin}\left\{\Phi_{k-2,2,0}\right\} .
$$

We remark that the method used here to select a basis of $T_{2, k}$ is not the only one. For $p=2$, it would be even simpler to consider the set $\left\{\Phi_{k, 0,2}, \Phi_{k-1,1,1}, \Phi_{k-2,2,0}\right\}$. It is clear from what we have done so far that these functionals provide a basis, with the understanding that trivial functionals are removed. However, the method described here has the advantage of being applicable for arbitrary rank $p$.

Now we consider valuations of rank $p=3$. For arbitrary $k \in \mathbb{N}_{0}$, we have

$$
\begin{aligned}
& T_{3, k}^{0}=\operatorname{lin}\left\{\Phi_{k, 0,3}, \Phi_{k-1,1,2}, \Phi_{k-2,2,1}, \Phi_{k-3,3,0}\right\}, \\
& T_{3, k}^{1}=\operatorname{lin}\left\{Q \Phi_{k-1,1,0}, Q \Phi_{k, 0,1}\right\}=\operatorname{lin}\left\{Q \Phi_{k-1,1,0}\right\} .
\end{aligned}
$$

The linear relation from (2.1) that corresponds to integers $k \in \mathbb{N}_{0}$ and $p=3$ is

$$
2 \pi \Phi_{k-2,2,1}+4 \pi \Phi_{k-1,1,2}+6 \pi \Phi_{k, 0,3}=Q \Phi_{k-1,1,0}+Q \Phi_{k, 0,1} .
$$

Let $k=0$ or $k=1$. Then $j_{0}=1$, and we have

$$
T_{3, k}^{0}=\operatorname{lin}\left\{\Phi_{k, 0,3}, \Phi_{k-1,1,2}\right\}
$$

as well as

Equation (4.2) states that

$$
T_{3, k}^{1}=\operatorname{lin}\left\{Q \Phi_{k-1,1,0}\right\}
$$

$$
4 \pi \Phi_{k-1,1,2}+6 \pi \Phi_{k, 0,3}=Q \Phi_{k-1,1,0} .
$$

Hence, $\left\{\Phi_{0,0,3}\right\}$ is a basis of $T_{3,0}$, because $T_{3,0}^{1}=\{0\}$. Further, a basis of $T_{3,1}$ is given by $\left\{\Phi_{1,0,3}, Q \Phi_{0,1,0}\right\}$.

Let $2 \leq k \leq n+1$. Then again $j_{0}=1$. Since $0 \leq k-2 \leq n-1$, the functional $\Phi_{k-2,2,1}$ on the left-hand side of (4.2) is nontrivial. In these cases, a basis of $T_{3, k}$ is precisely the set of nontrivial functionals belonging to $\left\{\Phi_{k, 0,3}, \Phi_{k-1,1,2}, \Phi_{k-3,3,0}, Q \Phi_{k-1,1,0}\right\}$. 
Finally, let $k=n+2$ or $k=n+3$. Then $j_{0}=0$ and $T_{3, k}=T_{3, k}^{0}=\operatorname{lin}\left\{\Phi_{k-3,3,0}\right\}$, because the other functionals are trivial. Therefore, $\left\{\Phi_{k-3,3,0}\right\}$ is a basis in these two cases.

\section{$\S 5$. Appendix}

In this section, we provide the construction of the special convex bodies whose existence was needed in the proof of Theorem 2.1. The crucial result, Lemma 5.3, is preceded by two preparatory lemmas.

Lemma 5.1. The polynomial

$$
P(x, y):=\alpha_{n} x^{n} y^{0}+\alpha_{n-1} x^{n-1} y^{1}+\ldots+\alpha_{0} x^{0} y^{n} \in \mathbb{R}[x, y]
$$

is a multiple of the polynomial $x^{2}+y^{2}$ if and only if

$$
\begin{aligned}
& \alpha_{1}-\alpha_{3}+\alpha_{5} \mp \ldots=0, \\
& \alpha_{0}-\alpha_{2}+\alpha_{4} \mp \ldots=0,
\end{aligned}
$$

where $\alpha_{i}:=0$ for $i \notin\{0, \ldots, n\}$.

Proof. Define the auxiliary polynomial

$$
\begin{aligned}
p(x, y):=\alpha_{n} x^{n-2} & +\alpha_{n-1} x^{n-3} y+\left(\alpha_{n-2}-\alpha_{n}\right) x^{n-4} y^{2}+\left(\alpha_{n-3}-\alpha_{n-1}\right) x^{n-5} y^{3} \\
& +\left(\alpha_{n-4}-\alpha_{n-2}+\alpha_{n}\right) x^{n-6} y^{4}+\left(\alpha_{n-5}-\alpha_{n-3}+\alpha_{n-1}\right) x^{n-7} y^{5}+\ldots \\
& +\left(\alpha_{3}-\alpha_{5}+\alpha_{7} \mp \ldots\right) x^{1} y^{n-3}+\left(\alpha_{2}-\alpha_{4}+\alpha_{6} \mp \ldots\right) y^{n-2} .
\end{aligned}
$$

Then we get

$$
\begin{aligned}
p(x, y)\left(x^{2}+y^{2}\right)=\alpha_{n} x^{n} & +\alpha_{n-1} x^{n-1} y^{1}+\ldots+\alpha_{2} x^{2} y^{n-2} \\
& +\left(\alpha_{3}-\alpha_{5} \pm \ldots\right) x y^{n-1}+\left(\alpha_{2}-\alpha_{4} \pm \ldots\right) y^{n} .
\end{aligned}
$$

Hence,

$$
P(x, y)-p(x, y)\left(x^{2}+y^{2}\right)=\left(\alpha_{1}-\alpha_{3}+\alpha_{5} \mp \ldots\right) x y^{n-1}+\left(\alpha_{0}-\alpha_{2}+\alpha_{4} \mp \ldots\right) y^{n},
$$

which implies the assertion of the lemma.

Next, we introduce a sequence of numbers which will be needed in the proof of Lemma 5.3. For $k \in \mathbb{N}_{0}$ and for odd numbers $r \in \mathbb{N}$ we define

$$
f_{k}(r):=\sum_{j=0}^{\frac{r-1}{2}}(-1)^{j} \frac{\Gamma\left(\frac{r-2 j+1}{2}\right)}{2^{2 j} j !(r-2 j) !}(k+1)^{j}
$$

and $f_{k}(-1):=0$.

Lemma 5.2. Let $r \in \mathbb{N}$ be odd, and let $k \in \mathbb{N}_{0}$. The numbers $f_{k}(r)$ satisfy the recursion

$$
2 r f_{k}(r)+k f_{k}(r-2)=\frac{(-1)^{\frac{r-1}{2}}(k+1)^{\frac{r-1}{2}}}{2^{r-2}\left(\frac{r-1}{2}\right) !}
$$

and can also be represented by

$$
f_{k}(r)=(-1)^{\frac{r-1}{2}} \sum_{j=0}^{\frac{r-1}{2}} \frac{(k+1)^{\frac{r-2 j-1}{2}} k^{j}}{2^{r-j-1}\left(\frac{r-2 j-1}{2}\right) ! r(r-2) \cdots(r-2 j)} .
$$


Proof. For the proof of the recursion, we write $r=2 m+1$ with $m \in \mathbb{N}_{0}$ and define

$$
\begin{aligned}
h_{k}(m, j) & :=(-1)^{j} \frac{\Gamma(m+1-j)}{2^{2 j} j !(2 m+1-2 j) !}(k+1)^{j} \text { for } j=0, \ldots, m, \\
h_{k}(m) & :=\sum_{j=0}^{m} h_{k}(m, j) .
\end{aligned}
$$

Then we have

$$
f_{k}(2 m+1)=h_{k}(m) .
$$

Zeilberger's algorithm (see [16]) produces the function

$$
g_{k}(m, j):=\frac{2 j}{-2 m-3+2 j} h_{k}(m, j)
$$

which satisfies the identity

$$
k h_{k}(m, j)+(4 m+6) h_{k}(m+1, j)=g_{k}(m, j+1)-g_{k}(m, j)
$$

for $j=0, \ldots, m-1$ and $m \geq 1$. This can be checked directly as follows. For $0 \leq j \leq$ $m-1$,

$$
\begin{aligned}
h_{k}(m+1, j) & =\frac{m+1-j}{(2 m+2-2 j)(2 m+3-2 j)} h_{k}(m, j) \\
& =\frac{1}{2(2 m+3-2 j)} h_{k}(m, j)
\end{aligned}
$$

and

$$
\begin{aligned}
h_{k}(m, j+1) & =-\frac{(k+1)(2 m-2 j)(2 m-2 j+1)}{(m-j) 2^{2}(j+1)} h_{k}(m, j) \\
& =-\frac{(k+1)(2 m-2 j+1)}{2(j+1)} h_{k}(m, j) .
\end{aligned}
$$

From (5.7) we deduce that

$$
g_{k}(m, j+1)=(k+1) h_{k}(m, j)
$$

and by (5.6) we thus obtain

$$
\begin{aligned}
k h_{k}(m, j) & +(4 m+6) h_{k}(m+1, j) \\
& =\frac{2 k m+3 k-2 k j+2 m+3}{2 m+3-2 j} h_{k}(m, j) \\
& =g_{k}(m, j+1)-g_{k}(m, j) .
\end{aligned}
$$

This confirms the required recursion (5.5) for $0 \leq j \leq m-1$ and $m \geq 1$.

Summing over $j=0, \ldots, m-1$, we get

$$
\begin{aligned}
g_{k}(m, m)-g_{k}(m, 0)= & k \sum_{j=0}^{m-1} h_{k}(m, j)+(4 m+6) \sum_{j=0}^{m-1} h_{k}(m+1, j) \\
= & k h_{k}(m)+(4 m+6) h_{k}(m+1)-k h_{k}(m, m) \\
& \quad-(4 m+6)\left(h_{k}(m+1, m)+h_{k}(m+1, m+1)\right)
\end{aligned}
$$


and from this,

$$
\begin{aligned}
k h_{k}(m)+ & (4 m+6) h_{k}(m+1) \\
= & g_{k}(m, m)-g_{k}(m, 0)+k h_{k}(m, m) \\
& \quad+(4 m+6)\left(h_{k}(m+1, m)+h_{k}(m+1, m+1)\right) \\
= & \frac{(-1)^{m+1}(k+1)^{m+1}}{2^{2 m+1}(m+1) !}
\end{aligned}
$$

which is also true for $m=0$. Here we used the special values

$$
\begin{aligned}
& g_{k}(m, 0)=0 \\
& g_{k}(m, m)=-\frac{2}{3} \frac{(k+1)^{m}}{\Gamma(m)(-4)^{m}}, \\
& h_{k}(m, m)=\frac{(k+1)^{m}}{m !(-4)^{m}} \\
& h_{k}(m+1, m)=\frac{1}{6} \frac{(k+1)^{m}}{m !(-4)^{m}}, \\
& h_{k}(m+1, m+1)=-\frac{1}{4} \frac{(k+1)^{m+1}}{(m+1) !(-4)^{m}},
\end{aligned}
$$

which are valid for all $m \in \mathbb{N}_{0}$. Now the recursion (5.3) follows immediately from (5.8).

We write (5.3) in the form

$$
f_{k}(r)=\frac{(-1)^{\frac{r-1}{2}}(k+1)^{\frac{r-1}{2}}}{2^{r-1}\left(\frac{r-1}{2}\right) ! r}-\frac{k}{2 r} f_{k}(r-2) .
$$

Equation (5.4) will be derived from (5.9) by induction. First, suppose that $r=1$. For any $k$, the definition of $f_{k}(r)$ implies that $f_{k}(1)=1$, which is consistent with (5.4). Assume that (5.4) has been proved for $r-2$ with some odd integer $r \geq 3$. We will establish (5.4) for the given number $r$. Relation (5.9) and the assumption imply that

$$
\begin{aligned}
f_{k}(r)= & \frac{(-1)^{\frac{r-1}{2}}(k+1)^{\frac{r-1}{2}}}{2^{r-1}\left(\frac{r-1}{2}\right) ! r} \\
& -\frac{k}{2 r}(-1)^{\frac{r-3}{2}} \sum_{j=0}^{\frac{r-3}{2}} \frac{(k+1)^{\frac{r-2 j-3}{2}} k^{j}}{2^{r-j-3}\left(\frac{r-2 j-3}{2}\right) !(r-2)(r-4) \cdots(r-2 j-2)} \\
= & \frac{(-1)^{\frac{r-1}{2}}(k+1)^{\frac{r-1}{2}}}{2^{r-1}\left(\frac{r-1}{2}\right) ! r} \\
& +\frac{k}{2 r}(-1)^{\frac{r-1}{2}} \sum_{j=1}^{\frac{r-1}{2}} \frac{(k+1)^{\frac{r-2 j-1}{2}} k^{j-1}}{2^{r-j-2}\left(\frac{r-2 j-1}{2}\right) !(r-2)(r-4) \cdots(r-2 j)} \\
= & \frac{(-1)^{\frac{r-1}{2}}(k+1)^{\frac{r-1}{2}}}{2^{r-1}\left(\frac{r-1}{2}\right) ! r}(-1)^{\frac{r-1}{2}} \sum_{j=1}^{\frac{r-1}{2}} \frac{(k+1)^{\frac{r-2 j-1}{2}} k^{j}}{\left(k+j-1\left(\frac{r-2 j-1}{2}\right) ! r(r-2) \cdots(r-2 j)\right.} \\
= & (-1)^{\frac{r-1}{2}} \sum_{j=0}^{\frac{r-1}{2}} \frac{(r-2 j-1}{2^{r-j-1}\left(\frac{r-2 j-1}{2}\right) ! r(r-2) \cdots(r-2 j)},
\end{aligned}
$$

which completes the induction and thus establishes (5.4). 
The main lemma yields the existence of a convex body $K$ for which the tensor $\Phi_{k, 0, s}(K)$, with $1 \leq k \leq n-1$ and $s \geq 2$, does not contain $Q$ as a factor, with respect to the symmetric tensor product. In the proof, we work with lower-dimensional simplexes. In the special case of a polytope $P$, explicit expressions for the Minkowski tensors are available; they result from the explicit representation of the support measures for polytopes. For $k<n$, we have

$$
\Phi_{k, 0, s}(P)=\frac{1}{s ! \omega_{n-k+s}} \sum_{F \in \mathcal{F}^{k}(P)} \mathcal{H}^{k}(F) \int_{N(P, F) \cap \mathbb{S}^{n-1}} u^{s} \mathcal{H}^{n-k-1}(\mathrm{~d} u),
$$

where $\mathcal{F}^{k}(P)$ denotes the set of $k$-dimensional faces of $P$ and $N(P, F)$ is the normal cone of $P$ at $F$. Let $E$ be a $j$-dimensional linear subspace of $\mathbb{R}^{n}(j<n)$, and let $P \subset E$ be a polytope. If $N_{E}(P, F)$ denotes the normal cone of $P$ at the face $F$ with respect to $E$, then

$$
\Phi_{k, 0, s}^{(E)}(P)=\frac{1}{s ! \omega_{j-k+s}} \sum_{F \in \mathcal{F}^{k}(P)} \mathcal{H}^{k}(F) \int_{N_{E}(P, F) \cap \mathbb{S}^{n-1}} u^{s} \mathcal{H}^{j-k-1}(\mathrm{~d} u)
$$

with $k<j$ is one of the Minkowski tensors of $P$, with $E$ as the reference space. The Minkowski tensors of $P$, evaluated in $\mathbb{R}^{n}$ on one side and in $E$ on the other, are related by formulas due to McMullen [14], namely,

$$
\Phi_{k, r, s}(K)=\sum_{m \geq 0} \frac{Q\left(E^{\perp}\right)^{m}}{(4 \pi)^{m} m !} \Phi_{k, r, s-2 m}^{(E)}(K)
$$

for $k \leq j$. This will be used below for the special case of simplexes.

Lemma 5.3. For $k, s \in \mathbb{N}$ with $1 \leq k \leq n-1$ and $s \geq 2$, there exists a convex body $K \in \mathcal{K}^{n}$ such that

for all $v \in \mathbb{T}^{s-2}$.

$$
\Phi_{k, 0, s}(K) \neq Q v
$$

Proof. Throughout the proof, let $\left\{e_{1}, \ldots, e_{n}\right\}$ be the standard basis of $\mathbb{R}^{n}$, and let $s \geq 2$.

We start with $n=2$, where only $k=1$ must be considered. For $t \in(0,1]$, we define triangles

$$
P_{t}:=\operatorname{conv}\left\{0, e_{1}, t e_{2}\right\} \subset \mathbb{R}^{2} .
$$

For the value of $\Phi_{1,0, s}$ at $P_{t}$, we get

$$
\begin{aligned}
\Phi_{1,0, s}\left(P_{t}\right) & =\sum_{F \in \mathcal{F}^{1}(P)} \frac{1}{s ! \omega_{s+1}} \mathcal{H}^{1}(F) \int_{N(P, F) \cap \mathbb{S}^{1}} u^{s} \mathcal{H}^{0}(\mathrm{~d} u) \\
& =\frac{1}{s ! \omega_{s+1}}\left(t\left(-e_{1}\right)^{s}+\left(-e_{2}\right)^{s}+\sqrt{1+t^{2}}\left(\frac{t e_{1}+e_{2}}{\sqrt{1+t^{2}}}\right)^{s}\right) \\
& =\frac{1}{s ! \omega_{s+1}}\left((-1)^{s} t e_{1}^{s}+(-1)^{s} e_{2}^{s}+\frac{1}{\sqrt{1+t^{2}}} \sum^{s-1} \sum_{j=0}^{s}\left(\begin{array}{l}
s \\
j
\end{array}\right) t^{j} e_{1}^{j} e_{2}^{s-j}\right) .
\end{aligned}
$$

Now we assume the existence of a tensor $v_{t} \in \mathbb{T}^{s-2}$ such that

$$
\Phi_{1,0, s}\left(P_{t}\right)=Q v_{t}
$$

is satisfied for all $t \in(0,1]$. Applying both sides of this equation to the $s$-tuple $(x, \ldots, x)$ with $x=\left(x_{1}, x_{2}\right) \in \mathbb{R}^{2}$ and using (5.11), we see that the polynomial

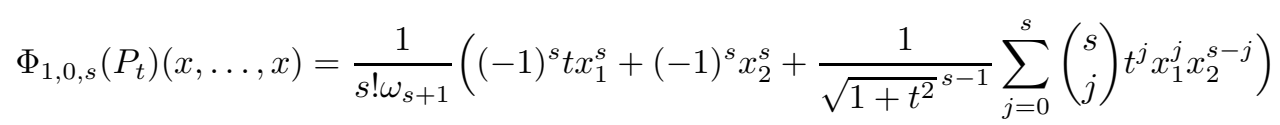


in $x_{1}, x_{2}$ is a multiple of $Q(x, x)=x_{1}^{2}+x_{2}^{2}$. Hence, Lemma 5.1 yields

$$
\begin{gathered}
0=(-1)^{s}+\frac{1}{{\sqrt{1+t^{2}}}^{s-1}}\left(\begin{array}{l}
s \\
0
\end{array}\right) t^{0}-\frac{1}{{\sqrt{1+t^{2}}}^{s-1}}\left(\begin{array}{l}
s \\
2
\end{array}\right) t^{2} \\
+\frac{1}{{\sqrt{1+t^{2}}}^{s-1}}\left(\begin{array}{l}
s \\
4
\end{array}\right) t^{4} \mp \ldots+\alpha(s) t
\end{gathered}
$$

for all $t \in(0,1]$ with

$$
\alpha(s)=\left\{\begin{aligned}
0 & \text { if } s \text { is odd } \\
1 & \text { if } s \equiv 0 \bmod 4 \\
-1 & \text { if } s \equiv 2 \bmod 4
\end{aligned}\right.
$$

We multiply (5.12) by ${\sqrt{1+t^{2}}}^{s-1}$ and see that (5.12) cannot be true for all $t \in(0,1]$ : for odd $s$, the resulting polynomial has nonzero highest coefficient, while for even $s$ it would follow that $\sqrt{1+t^{2}}$, multiplied by a nonzero polynomial, yields a polynomial. Thus, we have proved the assertion of the lemma in the case where $n=2$.

We turn to the case of general $n \geq 3$ and $k \in\{1, \ldots, n-1\}$. We define the $(k+1)$ simplex

$$
P^{(k)}:=\sqrt[k]{k !} \operatorname{conv}\left\{0, e_{1}, \ldots, e_{k+1}\right\} .
$$

It is contained in the $(k+1)$-dimensional linear subspace $L^{(k)}:=\operatorname{lin}\left\{e_{1}, \ldots, e_{k+1}\right\} \subset \mathbb{R}^{n}$. The $k$-faces of $P^{(k)}$ are

$$
F_{i}^{(k)}:=\sqrt[k]{k !} \operatorname{conv}\left\{0, e_{1}, \ldots, e_{i-1}, e_{i+1}, \ldots, e_{k+1}\right\},
$$

$i=1, \ldots, k+1$, and

$$
F_{k+2}^{(k)}:=\sqrt[k]{k !} \operatorname{conv}\left\{e_{1}, \ldots, e_{k+1}\right\} .
$$

For the $k$-dimensional volumes of these $k$-faces, we obtain

$$
\mathcal{H}^{k}\left(F_{i}^{(k)}\right)=1
$$

for $1 \leq i \leq k+1$, and

$$
\mathcal{H}^{k}\left(F_{k+2}^{(k)}\right)=\sqrt{k+1} .
$$

Using the comments preceding the statement of the lemma, we get

$$
\begin{aligned}
\Phi_{k, 0, s}^{\left(L^{(k)}\right)}\left(P^{(k)}\right) & =\frac{1}{s ! \omega_{s+1}}\left[\sum_{i=1}^{k+1} \mathcal{H}^{k}\left(F_{i}^{(k)}\right)\left(-e_{i}\right)^{s}+\mathcal{H}^{k}\left(F_{k+2}^{(k)}\right)\left(\frac{\sum_{i=1}^{k+1} e_{i}}{\sqrt{k+1}}\right)^{s}\right] \\
& =\frac{1}{s ! \omega_{s+1}}\left[\sum_{i=1}^{k+1}(-1)^{s} e_{i}^{s}+\frac{1}{\sqrt{k+1} s^{-1}}\left(\sum_{i=1}^{k+1} e_{i}\right)^{s}\right] .
\end{aligned}
$$

First, we treat the case of $k=n-1$. If $s$ is even, from

$$
\Phi_{n-1,0, s}\left(P^{(n-2)}\right)=\frac{1}{s ! \omega_{s+1}}\left(e_{n}^{s}+\left(-e_{n}\right)^{s}\right) \mathcal{H}^{n-1}\left(P^{(n-2)}\right)
$$

we conclude that the assertion of the lemma is true.

Now let $s$ be odd. Aiming at a contradiction, we assume the existence of some $v \in \mathbb{T}^{s-2}$ such that

$$
\Phi_{n-1,0, s}\left(P^{(n-1)}\right)=Q v .
$$

We apply both sides of this relation to the $s$-tuple $(x, \ldots, x)$ with $x=\left(x_{1}, 0, \ldots, 0, x_{n}\right) \in$ $\mathbb{R}^{n}$. Then the polynomial $\Phi_{n-1,0, s}\left(P^{(n-1)}\right)(x, \ldots, x)$ in the variables $x_{1}, x_{n}$ is a multiple 
of $Q(x, x)=x_{1}^{2}+x_{n}^{2}$. Observing that $\Phi_{n-1,0, s}^{\left(L^{(n-1)}\right)}=\Phi_{n-1,0, s}$, from (5.13) we deduce the formula

$$
\begin{aligned}
& \Phi_{n-1,0, s}\left(P^{(n-1)}\right)(x, \ldots, x) \\
& \quad=\frac{1}{s ! \omega_{s+1}}\left((-1)^{s} x_{1}^{s}+(-1)^{s} x_{n}^{s}+\frac{1}{\sqrt{n}^{s-1}}\left(x_{1}+x_{n}\right)^{s}\right) \\
& \quad=\frac{1}{s ! \omega_{s+1}}\left((-1)^{s} x_{1}^{s}+(-1)^{s} x_{n}^{s}+\frac{1}{\sqrt{n}^{s-1}} \sum_{i=0}^{s}\left(\begin{array}{l}
s \\
i
\end{array}\right) x_{1}^{i} x_{n}^{s-i}\right) .
\end{aligned}
$$

We view the right-hand side as a polynomial in $x_{1}, x_{n}$ and apply Lemma 5.1. Since $s$ is odd, we obtain

$$
0=(-1)^{s}+\frac{1}{\sqrt{n}^{s-1}}\left(\begin{array}{l}
s \\
0
\end{array}\right)-\frac{1}{\sqrt{n}^{s-1}}\left(\begin{array}{l}
s \\
2
\end{array}\right)+\frac{1}{\sqrt{n}^{s-1}}\left(\begin{array}{l}
s \\
4
\end{array}\right) \mp \ldots
$$

This is equivalent to

$$
n^{\frac{s-1}{2}}=\left(\begin{array}{l}
s \\
0
\end{array}\right)-\left(\begin{array}{l}
s \\
2
\end{array}\right)+\left(\begin{array}{l}
s \\
4
\end{array}\right) \mp \ldots=\operatorname{Re}\left((i+1)^{s}\right)= \pm 2^{\frac{s-1}{2}},
$$

which is a contradiction for $n>2$ and $s \geq 2$. This settles the case where $k=n-1$ in the lemma.

Finally, we consider the cases where $k<n-1$. Let $s \geq 2$ be an arbitrary integer. A special case of (5.10) gives

$$
\Phi_{k, 0, s}\left(P^{(k)}\right)=\sum_{j \geq 0} \frac{Q\left(\left(L^{(k)}\right)^{\perp}\right)^{j}}{(4 \pi)^{j} j !} \Phi_{k, 0, s-2 j}^{\left(L^{(k)}\right)}\left(P^{(k)}\right) .
$$

We apply both sides to the $s$-tuple $(x, \ldots, x)$ with $x=\left(x_{1}, 0, \ldots, 0, x_{n}\right) \in \mathbb{R}^{n}$. Using (5.13), we obtain

$$
\Phi_{k, 0, s}\left(P^{(k)}\right)(x, \ldots, x)=\sum_{j \geq 0} \frac{x_{n}^{2 j}}{(4 \pi)^{j} j !(s-2 j) ! \omega_{s-2 j+1}}\left((-1)^{s-2 j} x_{1}^{s-2 j}+\frac{x_{1}^{s-2 j}}{\sqrt{k+1}^{s-2 j-1}}\right) .
$$

Here and in the following, summation with respect to $j$ is restricted to $j \leq s / 2$. In order to arrive at a contradition, we again assume that the left-hand side is a multiple of $x_{1}^{2}+x_{n}^{2}$. Then Lemma 5.1 implies that

$$
\sum_{j \geq 0}(-1)^{j} \frac{1}{(4 \pi)^{j} j !(s-2 j) ! \omega_{s-2 j+1}}\left((-1)^{s-2 j}+\frac{1}{\sqrt{k+1}^{s-2 j-1}}\right)=0 .
$$

Using

$$
\omega_{s-2 j+1}=\frac{2 \pi^{\frac{s-2 j+1}{2}}}{\Gamma\left(\frac{s-2 j+1}{2}\right)}
$$

we find that (5.15) is equivalent to

$$
\sum_{j \geq 0}(-1)^{j} \frac{\Gamma\left(\frac{s-2 j+1}{2}\right)}{2^{2 j} j !(s-2 j) !}\left((-1)^{s} \sqrt{k+1}^{s-1}+\sqrt{k+1}^{2 j}\right)=0 .
$$

First, we consider the case where $s$ is even. Since

$$
\Gamma\left(\frac{s}{2}-j+\frac{1}{2}\right)=\frac{(s-2 j) ! \sqrt{\pi}}{\left(\frac{s}{2}-j\right) ! 2^{s-2 j}}
$$


we get

$$
\begin{aligned}
& \sum_{j=0}^{\frac{s}{2}}(-1)^{j} \frac{\Gamma\left(\frac{s-2 j+1}{2}\right)}{2^{2 j} j !(s-2 j) !}\left(\sqrt{k+1}^{s-1}+\sqrt{k+1}^{2 j}\right) \\
& =\sum_{j=0}^{\frac{s}{2}}(-1)^{j} \frac{(s-2 j) ! \sqrt{\pi}}{\left(\frac{s}{2}-j\right) ! 2^{s-2 j} 2^{2 j} j !(s-2 j) !}\left(\sqrt{k+1}^{s-1}+\sqrt{k+1}^{2 j}\right) \\
& =\frac{\sqrt{\pi}}{\left(\frac{s}{2}\right) ! 2^{s}} \sum_{j=0}^{\frac{s}{2}}(-1)^{j} \frac{\left(\frac{s}{2}\right) !}{\left(\frac{s}{2}-j\right) ! j !}\left(\sqrt{k+1}^{-1}+\sqrt{k+1}^{2 j}\right) \\
& =\frac{\sqrt{\pi}}{\left(\frac{s}{2}\right) ! 2^{s}}\left[((-1)+1)^{\frac{s}{2}} \sqrt{k+1} s-1+(-(k+1)+1)^{\frac{s}{2}}\right] \\
& =\frac{\sqrt{\pi}}{\left(\frac{s}{2}\right) ! 2^{s}}(-k)^{\frac{s}{2}} \neq 0 .
\end{aligned}
$$

This yields the required contradiction if $s$ is even.

Next, we address the case of an odd integer $s$. For $l \in \mathbb{N}_{0}$, we put

$$
f_{l}(s):=\sum_{j=0}^{\frac{s-1}{2}}(-1)^{j} \frac{\Gamma\left(\frac{s-2 j+1}{2}\right)}{2^{2 j} j !(s-2 j) !}(l+1)^{j} .
$$

Then (5.16) can be rewritten as

$$
-(k+1)^{\frac{s-1}{2}} f_{0}(s)+f_{k}(s)=0 .
$$

By Lemma 5.2, $f_{l}$ satisfies the recursion

$$
2 s f_{l}(s)+l f_{l}(s-2)=\frac{(-1)^{\frac{s-1}{2}}(l+1)^{\frac{s-1}{2}}}{2^{s-2}\left(\frac{s-1}{2}\right) !},
$$

that is,

$$
f_{l}(s)=\frac{(-1)^{\frac{s-1}{2}}(l+1)^{\frac{s-1}{2}}}{2^{s-1}\left(\frac{s-1}{2}\right) ! s}-\frac{l}{2 s} f_{l}(s-2)
$$

and especially

$$
f_{0}(s)=\frac{(-1)^{\frac{s-1}{2}}}{2^{s-1}\left(\frac{s-1}{2}\right) ! s} .
$$

Substituting (5.18) and (5.19) into (5.17), we obtain

$$
f_{k}(s-2)=0 .
$$

This is a contradiction to Lemma 5.2, which yields for $f_{k}(s-2)$ an explicit value different from zero. Thus, for all $s \geq 2$ and $n-1>k \geq 1$, equation (5.16) cannot be satisfied, which contradicts our assumption. This proves the assertion of the lemma also for $k<n-1$.

\section{REFERENCES}

[1] S. Alesker, Continuous valuations on convex sets, Geom. Funct. Anal. 8 (1998), 402-409. MR1616167 (99b:52027)

[2] _ Continuous rotation invariant valuations on convex sets, Ann. of Math. (2) 149 (1999), 977-1005. MR1709308 (2000i:52019)

[3] - Description of continuous isometry covariant valuations on convex sets, Geom. Dedicata 74 (1999), 241-248. MR1669363 (2000a:52026)

[4] _ On P. McMullen's conjecture on translation invariant valuations, Adv. Math. 155 (2000), 239-263. MR1794712 (2001k:52013) 
[5] - Description of translation invariant valuations on convex sets with solution of P. McMullen's conjecture, Geom. Funct. Anal. 11 (2001), 244-272. MR1837364 (2002e:52015)

[6] - Hard Lefschetz theorem for valuations, complex integral geometry, and unitarily invariant valuations, J. Differential Geom. 63 (2003), 63-95. MR2015260 (2004h:52015)

[7] — SU(2)-invariant valuations, Geometric Aspects of Functional Analysis, Lecture Notes in Math., vol. 1850, Springer, Berlin, 2004, pp. 21-29. MR2087147 (2005f:52029)

[8] C. Beisbart, M. S. Barbosa, H. Wagner, and L. da F. Costa, Extended morphometric analysis of neuronal cells with Minkowski valuations, arXiv:cond-mat/0507648.

[9] C. Beisbart, R. Dahlke, K. Mecke, and H. Wagner, Vector- and tensor-valued descriptors for spatial patterns, Morphology of Condensed Matter, Lecture Notes in Phys., vol. 600, Springer, Berlin, 2002, pp. 238-260.

[10] H. Hadwiger and R. Schneider, Vektorielle Integralgeometrie, Elem. Math. 26 (1971), 49-57. MR0283737 (44:967)

[11] D. A. Klain, A short proof of Hadwiger's characterization theorem, Mathematika 42 (1995), 329339. MR1376731 (97e:52008)

[12] P. McMullen, Continuous translation-invariant valuations on the space of compact convex sets, Arch. Math. (Basel) 34 (1980), 377-384. MR0593954 (81m:52013)

[13] _ Valuations and dissections, Handbook of Convex Geometry, Vols. A, B, Elsevier, Amsterdam, 1993, pp. 933-988. MR1243000 (95f:52018)

[14] - Isometry covariant valuations on convex bodies, Rend. Circ. Mat. Palermo (2) Suppl. 50 (1997), 259-271. MR1602986 (99a:52016)

[15] P. McMullen and R. Schneider, Valuations on convex bodies, Convexity and its Applications, Birkhäuser, Basel, 1983, pp. 170-247. MR0731112 (85e:52001)

[16] M. Petkovsek, H. Wilf, and D. Zeilberger, $A=B$, A K Peters, Ltd., Wellesley, MA, 1996. MR1379802 (97j:05001)

[17] A. V. Pukhlikov and A. G. Khovanskiǔ, Finitely-additive measures of virtual polytopes, Algebra i Analiz 4 (1992), no. 2, 161-185; English transl., St. Petersburg Math. J. 4 (1993), no. 2, 337-356. MR1182399 (93i:52024)

[18] R. Schneider, On Steiner points of convex bodies, Israel J. Math. 9 (1971), 241-249. MR0278187 $(43: 3918)$

[19] _ Krümmungsschwerpunkte konvexer Körper. I, II, Abh. Math. Sem. Univ. Hamburg 37 (1972), 112-132, 204-217. MR0307039 (46:6160); MR0331220 (48:9554)

[20] Convex bodies: the Brunn-Minkowski theory, Encyclopedia Math. Appl., vol. 44, Cambridge Univ. Press, Cambridge, 1993. MR1216521 (94d:52007)

[21] - Tensor valuations on convex bodies and integral geometry, Rend. Circ. Mat. Palermo (2) Suppl. 65, Part 1 (2000), 295-316. MR1809137 (2002c:52013)

[22] R. Schneider and R. Schuster, Tensor valuations on convex bodies and integral geometry. II, Rend. Circ. Mat. Palermo (2) Suppl. 70, Part 2 (2002), 295-314. MR1962603 (2004c:52011)

Mathematisches Institut, Albert-Ludwigs-Universität, D-79104 Freiburg i. Br., Germany E-mail address: daniel.hug@math.uni-freiburg.de

Mathematisches Institut, Albert-Ludwigs-Universität, D-79104 Freiburg i. Br., Germany E-mail address: rolf.schneider@math.uni-freiburg.de

Mathematisches Institut, Albert-Ludwigs-Universität, D-79104 Freiburg i. Br., Germany E-mail address: raschuster@munichre.com

Received 1/AUG/2006

Originally published in English 